\title{
Intraoperative Changes in Cerebrospinal Fluid Gas Tensions Reflect Paraplegia During Thoracoabdominal Aortic Surgery: A Proof-of-Principle Study
}

Vascular and Endovascular Surgery

2015, Vol. 49(3-4) 84-92

(C) The Author(s) 2015

Reprints and permission:

sagepub.com/journalsPermissions.nav DOI: I0.II77/I5385744I5595210

ves.sagepub.com

(S)SAGE

\author{
Felix Fleissner, MD', Ahmed Redwan, MD', \\ Theodosios Bisdas, MD', Anna-Lena Boeck, MD², \\ Karin Weissenborn, $\mathbf{M D}^{2}$, Axel Haverich, MD', \\ Omke E. Teebken, MD', Maximilian Pichlmaier, MA, MD ${ }^{1,3}$, \\ and Andreas Martens, MD'
}

\begin{abstract}
Background: In this study, gas tensions in cerebrospinal fluid (CSF) were prospectively evaluated as intraoperative markers for the detection of neurological deficits. Methods: Spinal fluid, serum, and heart lung machine (HLM) perfusate were monitored for gas tensions $\left(\mathrm{PO}_{2} / \mathrm{PCO}_{2}\right)$ and related parameters $(\mathrm{pH}$, lactate, and glucose) during thoracoabdominal aortic repair and correlated with perioperative neurological examination and electrophysiological testing. Results: Forty-seven patients were assessed for the study, and 40 consecutive patients were finally included. The patients were divided into 3 groups: group A ( 23 patients, $57.5 \%$ ): no clinical or laboratory signs of neurological damage; group B (14 patients, $35 \%$ ) who developed subclinical deficits; and group C ( 3 patients, 7.5\%) who had paraplegia. Significant intraoperative changes in CSF gas tensions were observed with postoperative paraplegia. Glucose ratio between serum and CSF showed higher variability in group C, confirming a damage of the blood-brain barrier (BBB). Conclusion: Major neurological damage is reflected by early changes in CSF gas tensions and glucose variability, suggesting damage of the $B B B$ in these patients.
\end{abstract}

\section{Keywords}

thoracoabdominal aortic repair, CSF drainage, paraplegia

\section{Introduction}

Despite vast improvements throughout the past 20 years, thoracoabdominal aortic repair (TAAA repair) still bears a high risk in terms of mortality and morbidity. ${ }^{1,2}$ The most devastating complication is paraplegia as a consequence of intraoperative or early postoperative spinal cord ischemia. ${ }^{3-5}$ Numerous so-called adjuncts to the original clamp-and-sew technique have reduced the rate of paraplegia and paraparesis from as much as $20 \%$ to $30 \%$ to below $15 \%$. ${ }^{6,7}$ The widely accepted 3 most relevant adjuncts are (1) sequential clamping, (2) distal perfusion (DP), and (3) cerebrospinal fluid (CSF) drainage. ${ }^{1-7}$

Intraoperative monitoring of spinal cord function, in order to adjust the procedure accordingly, has been reported by some groups ${ }^{8,9-13}$ to be feasible. In this series, the hypothesis was tested that CSF, which passes the critical part of the spinal cord in a craniocaudal direction, ${ }^{14,15}$ may reflect short-term changes in the gas exchange, given that especially $\mathrm{CO}_{2}$ is highly soluble and traverses semipermeable membranes easily. The $\mathrm{pO}_{2}$ in the absence of hemoglobin and
$\mathrm{pCO}_{2}$ are determined by a comparatively small number of molecules and should, thus, reflect very small changes of their concentration in the tissue embedded in CSF- the spinal cord. Similarly, one would expect $\mathrm{pH}$ and lactate to signal anaerobic metabolism and glucose ratio between serum and CSF to reflect damage of blood-brain barrier (BBB). ${ }^{8}$

The aim of our proof-of-principle study was to assess whether changes in CSF gas tensions might reflect neurological outcome measured by changes in somatosensory evoked

\footnotetext{
'Department of Cardiac, Thoracic, Transplantation and Vascular Surgery, Hannover Medical School, Hannover, Germany

${ }^{2}$ Department of Neurology, Hannover Medical School, Hannover, Germany

${ }^{3}$ Department of Cardiac Surgery, University Hospital Munich, Ludwig-Maximilian-University, Munich
}

\section{Corresponding Author:}

Felix Fleissner, Department of Cardiac, Thoracic, Transplantation and Vascular Surgery, Hanover Medical School, Carl-Neuberg Str I, D-30625, Hannover, Germany.

Email: fleissner.felix@mh-hannover.de 
Table I. Patients' Demographics, Comorbidities, and Characteristics.

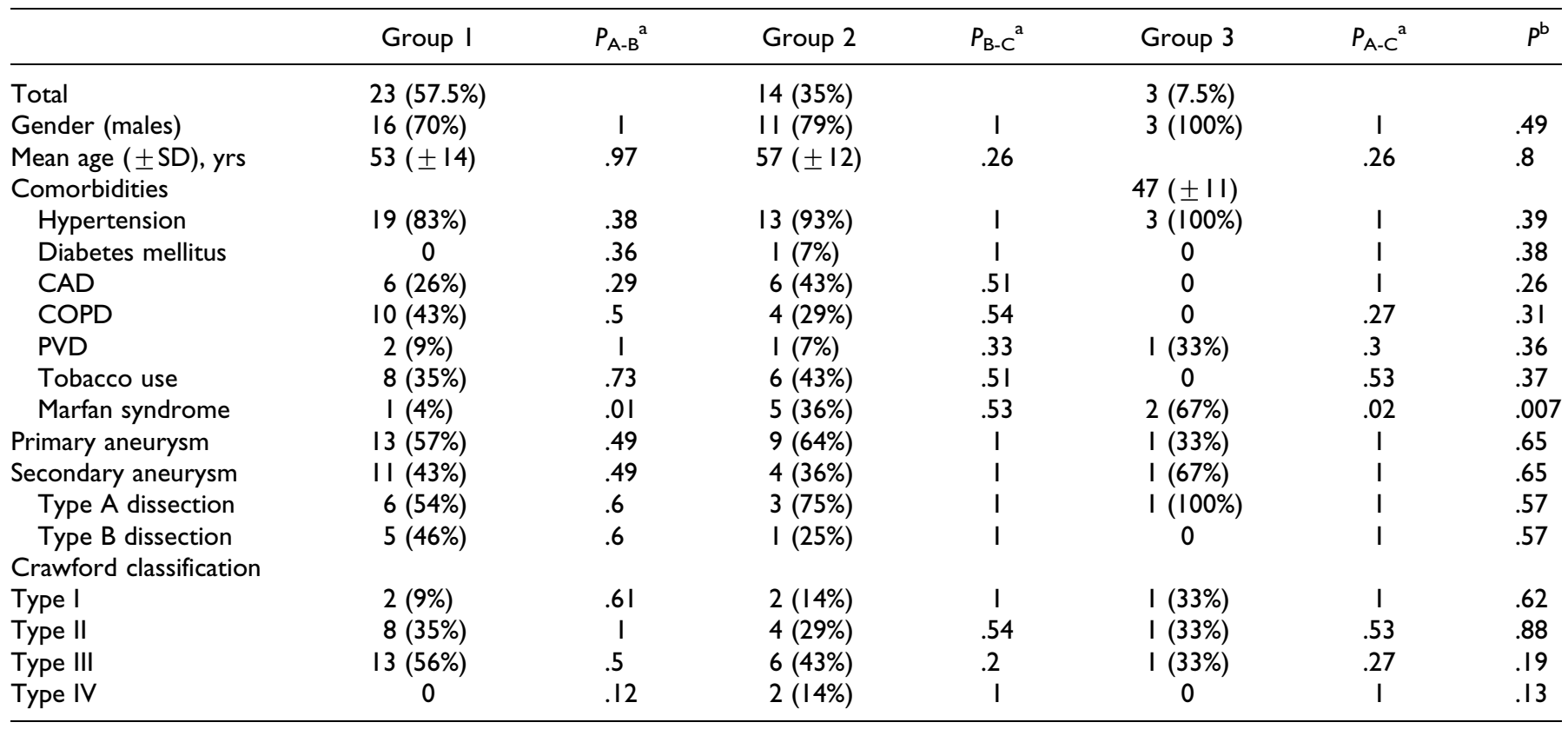

Abbreviations: SD, standard deviation; CAD, coronary artery disease; COPD, chronic obstructive pulmonary disease; PVD, peripheral vascular disease; ANOVA, analysis of variance.

${ }^{\text {a }} P_{X-Y}$ : group $X$ versus group $Y$.

${ }^{b}$ Comparison between 3 groups using one-way ANOVA or Kruskal-Wallis test.

potential/motor evoked potential (SEP/MEP) or as clinically apparent paraplegia.

\section{Patients and Methods}

Between March 2008 and April 2011, 47 patients agreed to be included in our study. The institutional review board approved this study. All patients gave written informed consent in accordance with the Declaration of Helsinki. Patients' demographics, comorbidities, and characteristics are listed in Table 1.

\section{Neurological Testing}

Electrophysiological testing (SEP/MEP) was performed in the neurology laboratory within 2 days preoperatively. The patients were examined by 1 of the 2 neurology coauthors. This was repeated as soon as the patients returned from the intensive care unit to the normal ward on days 4 to 7 .

\section{Analysis of CSF}

Spinal cord drainage was performed in all patients. A CSF catheter (Integra Neurosciences, Plainsboro, New Jersey) was placed the evening before surgery. Insertion site of the catheter was L3 to L4 or L2 to L3, and the catheter tip was positioned approximately at T9 to T10. The catheter was connected to an external drainage system (Lumbar Catheter Accessory Kit; Integra Neurosciences), allowing CSF drainage as well as continuous pressure monitoring.
Samples of CSF for semionline gas analysis (within 2 minutes) were taken in 15-minute intervals beginning just prior to starting DP and continuing to 2 hours thereafter. At the same time, interval blood samples were drawn from the right radial artery and the arterial line of the heart lung machine and also entered for gas analysis. Parameters evaluated were $\mathrm{po}_{2}, \mathrm{pCO}_{2}$, $\mathrm{pH}$, lactate, and glucose.

\section{Operative Technique}

The operative procedure and the anesthesiological management were highly standardized and have been described elsewhere. $^{1,8}$ In all patients, DP was achieved using a minicircuit cardiopulmonary bypass (CPB; Terumo, Tokyo, Japan, ROC-Safe) connected via cannulation of left femoral artery and vein. Venous access was obtained percutaneously using a $22 \mathrm{~F}$ 2-stage cannula for venous drainage (FemTrack 20-24F; Edwards Lifesciences, Irvine, USA). The left femoral artery was exposed through a small transverse skin incision and a $15 \mathrm{~F}$ or $17 \mathrm{~F}$ cannula introduced nonocclusively by puncture through a small purse-string suture in the adventitia (Novalung, Novaport KU-15/17F; Hechingen, Germany). The DP pressures, as measured in the right femoral artery, were kept at $70 \mathrm{~mm} \mathrm{Hg}$ or above, with a minimal pump flow of $1.5 \mathrm{~L} / \mathrm{min}$. Proximal systolic pressures, determined in the right radial artery, were kept above $120 \mathrm{~mm} \mathrm{Hg}$. Selective organ perfusion was additionally used for the renal and visceral arteries during the time the relevant aortic segment was clamped (staged clamping). Heparin dosing for this series was set at a 
conservative $300 \mathrm{IU} \mathrm{kg}^{-1}$ aiming for an ACT $>200$ seconds. During clamping, the average temperature (rectal vs oesophageal) was kept around $33^{\circ} \mathrm{C}$ and rewarming started immediately on declamping. The temperature was chosen as a compromise between improved organ protection by lower temperatures and the increased risk of spontaneous ventricular fibrillation below $32^{\circ} \mathrm{C}$.

\section{Cerebrospinal Fluid Drainage}

The CSF pressures were recorded continuously and CSF drained at intervals whenever values exceeded 12 to $15 \mathrm{~mm}$ $\mathrm{Hg}$ into the collection system.

\section{Neurological Evaluation}

An experienced neurologist examined all patients prior to and as soon as possible after the operation. In patients with postoperative confusional states, cranial computed tomography scanning was performed to exclude cerebral pathology. The modified Tarlov scoring scale was applied to assess lower limb motor function. Patients underwent detailed electrophysiological evaluation using SEP and MEP prior to and after the surgery. The postoperative evaluation was performed 4 to 7 days after surgery. All new pathologic SEP and MEP were considered neurological deficits and the patients included in the corresponding group for evaluation.

\section{Study Design and Definitions}

The study was performed prospectively. Patients undergoing postoperative neurologic examination were divided into 3 groups, regarding the findings of postoperative neurological and electrophysiology examinations. The first group showed no clinical or laboratory signs of neurological complications (group A, 29 of 45 patients; 64\%). In the second group (group B, 13 of 40 patients, 29\%), all patients with pathological SEP or MEP and/or subclinical signs of neurological deficit were included (Table 2). Subclinical in this context was defined as any pathological neurological finding that was not obvious or reported by the patient and did not significantly interfere with postoperative mobilization and physiotherapy. Finally, patients having an obvious severe postoperative deficit such as paraplegia or paraparesis were included in a third group (group C, 3 patients of 45; 7\%). The groups were compared in terms of comorbidities, characteristics, and operative details in order to exclude any undue difference that may introduce bias.

One patients, without clinical signs of neurological deficit, denied any postoperative neurological examination, and 6 patients died without any neurological examination. These individuals were excluded from this study. Of the patients analyzed for neurological symptoms, 3 patients in group A and 1 patient in group $\mathrm{C}$ died. One patient in group $\mathrm{C}$ had a, most likely, thrombotic occlusion of the left femoral artery which required subsequent multiple revisions of the femoral
Table 2. Clinical, Subclinical, and Laboratory (SEP and MEP) Findings in Patients of Groups B and C.

\begin{tabular}{|c|c|c|}
\hline Patients & SEP and MEP Examination & Clinical Examination \\
\hline \multicolumn{3}{|l|}{ Group B } \\
\hline Patient I & $\begin{array}{l}\text { Amplitude reduction } \\
\text { (MEP) on both sides } \\
\text { (OBS) and amplitude } \\
\text { prolongation (SEP) on } \\
\text { right side (RS) }\end{array}$ & Normal \\
\hline Patient 2 & Not assessed & $\begin{array}{l}\text { Left hemiparesis and } \\
\text { hypaesthesia }^{\mathrm{a}}\end{array}$ \\
\hline Patient $3^{b}$ & $\begin{array}{l}\text { Amplitude prolongation } \\
\text { (SEP) on left side (LS) } \\
\text { and amplitude } \\
\text { reduction (SEP) on RS }\end{array}$ & Normal \\
\hline Patient 4 & Not assessed & $\begin{array}{l}\text { Transient left leg } \\
\text { monoparesis }\end{array}$ \\
\hline Patient 5 & $\begin{array}{l}\text { Amplitude prolongation } \\
\text { (SEP) on LS. MEP: } \\
\text { normal }\end{array}$ & $\begin{array}{l}\text { Transient paresthesia of } \\
\text { left lower limb (tingle- } \\
\text { like). Light (central) left } \\
\text { leg paresis }\end{array}$ \\
\hline Patient 6 & Not assessed & Normal \\
\hline Patient 7 & $\begin{array}{l}\text { Amplitude prolongation } \\
\text { (SEP) on LS and }\end{array}$ & $\begin{array}{l}\text { Suspect positive Babinski } \\
\text { reflex }\end{array}$ \\
\hline
\end{tabular}

Patient 9 Amplitude prolongation Normal

(SEP) on LS and amplitude reduction (MEP) on LS

Patient 10 Amplitude prolongation (SEP) on LS and amplitude reduction (MEP) on RS

Patient II Amplitude prolongation (SEP) on LS

Patient 12 Not assessed

Normal

Patient 13 Amplitude reduction (SEP) Normal on LS

Patient 14 Amplitude reduction (SEP) Normal on both sides (OBS) and amplitude prolongation (MEP) on RS

Group C

Patient I Not assessed Paraplegia

Patient 2 Not assessed Paraplegia

Patient 3 Not assessed Paraplegia

Abbreviations: CT, computed tomography; SEP, somatosensory evoked potential; MEP, motor evoked potential.

antraoperative cardiac resuscitation due to ventricular fibrillation; the cranial CT scan was normal.

${ }^{\mathrm{b}}$ The patient rejected the MEP examination postoperatively.

artery. He had a compartment syndrome, renal insufficiency, and pneumonia during the postoperative course. The patient developed a sepsis and died due to multiorgan failure, despite a rescue attempt using extracorporeal life support. One patient in group A developed a fulminant pancreatitis and died due to multiorgan failure. The other patient in group 
A developed a severe cerebral herniation during postoperative course due to massive coagulation disorder after reoperation due to a hemathothorax. The third patient of group A died during rehabilitation due to sudden cardiac death. The patient had a known history of dilatative cardiomyopathy, however, the exact cause of death remains unclear since no autopsy was performed.

Serum-, CSF- as well as CPB samples were collected before aortic clamping and every 15 minutes thereafter until the end of surgery. All samples were immediately analyzed intraoperatively for $\mathrm{pO}_{2}, \mathrm{pCo}_{2}, \mathrm{pH}$, lactate, and glucose levels. The measurements were performed on a Hitachi (Hitachi Ltd. Tokyo, Japan) automated clinical analyzer with enzymatic in vitro tests. The validity of the measurements with CSF samples had been confirmed in a prior extensive test series.

We compared all measured values between the 3 groups for each point in time. Besides the individual measurements, correlations between the specific parameters $\left(\mathrm{pCO}_{2}\right.$ and $\left.\mathrm{po}_{2}\right)$ in serum, CPB, and CSF were looked at for evidence of significant contribution of DP to the oxygenation of the CSF. Finally, variability of the ratio between glucose levels in serum and CSF was compared between the groups, in order to assess any damage of blood-brain barrier (BBB).

\section{Statistical Methods}

All analyses and graphs were performed with GraphPad Software Inc Version 5.0. Categorical variables and frequencies are presented as percentages, and continuous variables as mean \pm standard deviation (SD). D' Agostino and Pearson omnibus normality test determined the distribution of continuous variables. Ordinal data were compared with Fisher exact test. Normally distributed continuous variables were compared with $t$ test for independent variables, and MannWhitney $U$ test was performed for not normally distributed data. Within-group changes regarding the obtained parameters were analyzed by repeated measures analysis of variance (ANOVA). Between-group differences at the different time points were analyzed by one-way ANOVA for continuous variables and by Kruskal-Wallis test for nonparametric data. Finally, comparison of SD (glucose variability) was performed by means of the $F$ test. The Pearson correlation coefficient was measured in order to analyze the correlation between the gas tensions $\left(\mathrm{po}_{2}\right.$ and $\left.\mathrm{pCo}_{2}\right)$ of serum, $\mathrm{CSF}$, and DP. The $P$ value of $\leq .05$ was considered statistically significant for individual tests. Where data points were missing, the number of available observations is reported, and no assumptions are made about missing data.

\section{Results}

The mean duration of the operation was $316 \pm 81$ minutes in group A, $263 \pm 55$ minutes in group $\mathrm{B}$, and $430 \pm 150$ minutes in group $\mathrm{C}(P=0.103)$, respectively. The DP time was also comparable between the 3 groups (group A: $141 \pm 44$ minutes, group B: $122 \pm 36$, and group C: $171 \pm 78$ minutes,
$P=.28)$. The selective organ perfusion was $67 \pm 40$ minutes in group A, $65 \pm 43$ minutes in group $\mathrm{B}$, and $76 \pm 99$ minutes in group $\mathrm{C}(P=.66)$.

\section{Cerebrospinal Fluid}

The examined markers in the CSF samples showed no statistically significant differences between groups A and B. In contrast, the comparison between groups $\mathrm{A}$ and $\mathrm{C}$ yielded statistically significant differences at different time points during the aortic clamping.

The $\mathrm{pO}_{2}$ in CSF was found to be remarkably constant in all groups throughout the operation in contrast to serum (Figure 1). Noteworthy is that 45 and 120 minutes after aortic clamping, we found a statistically significant decrease of $\mathrm{po}_{2}$ in group $\mathrm{C}$ in comparison to the other groups (Figure 1).

The $\mathrm{pCO}_{2}$ in CSF (similarly to serum) was constant for all groups (Figure 2). Patients in group $\mathrm{C}$ showed the highest $\mathrm{pCo}_{2}$ levels 15 minutes after aortic clamping, a trend that lasted until declamping of the aorta.

$\mathrm{pH}$ was found higher than expected in group A and especially in group B, whereas it was expectedly lower in group C (Table 3). Lactate, similarly, was found higher in group C than in both other groups starting 30 minutes following aortic clamping.

Finally, glucose levels were much higher in group C starting after 60 minutes of aortic clamping (Figure 3 ). The statistical comparison of the standard deviations ( $F$ test) of glucose ratio between serum and CSF showed statistically significant higher variability in group $\mathrm{C}$ after $45(F: 4.51, P=.04), 60$ $(F: 4.37, P=.04), 105$ ( $F: 5.76, P=.04)$, and 120 minutes $(F: 36, P<.001)$.

\section{Distal Perfusion}

In order to exclude bias contributed by the levels of each parameter in the blood coming directly from the mini-circuit, we compared all reported parameters between the DP samples of each group, without statistically significant differences.

\section{Correlation of $\mathrm{pO}_{2}$ and $\mathrm{pCO}_{2}$}

The $\mathrm{po}_{2}$ in serum and CSF showed no statistically significant correlation ( $r: .02, P=.73,95 \% \mathrm{CI}:-0.10$ to 0.14$)$. In contrast, we found a relationship between $\mathrm{po}_{2(\mathrm{DP})}$ and $\mathrm{po}_{2(\mathrm{CSF})}(r: .16$, $P=.009$, 95\% CI: 0.04 to 0.28 ) (See Figure 4). Similar was the behavior of $\mathrm{pCO}_{2}$ showing no relationship between CSF and serum ( $r: .07, P=.22,95 \% \mathrm{CI}:-0.04$ to 0.19$)$ but a positive relationship between CSF and DP $(r: 0.20, P=.001,95 \%$ CI: 0.08 to 0.32 ).

\section{Serum}

Serum samples of all measured parameters showed a considerable variability within the physiological ranges. No statistically significant differences between the groups regarding $\mathrm{pO}_{2}, \mathrm{pCO}_{2}$, $\mathrm{pH}$, glucose, and lactate levels were demonstrated. 


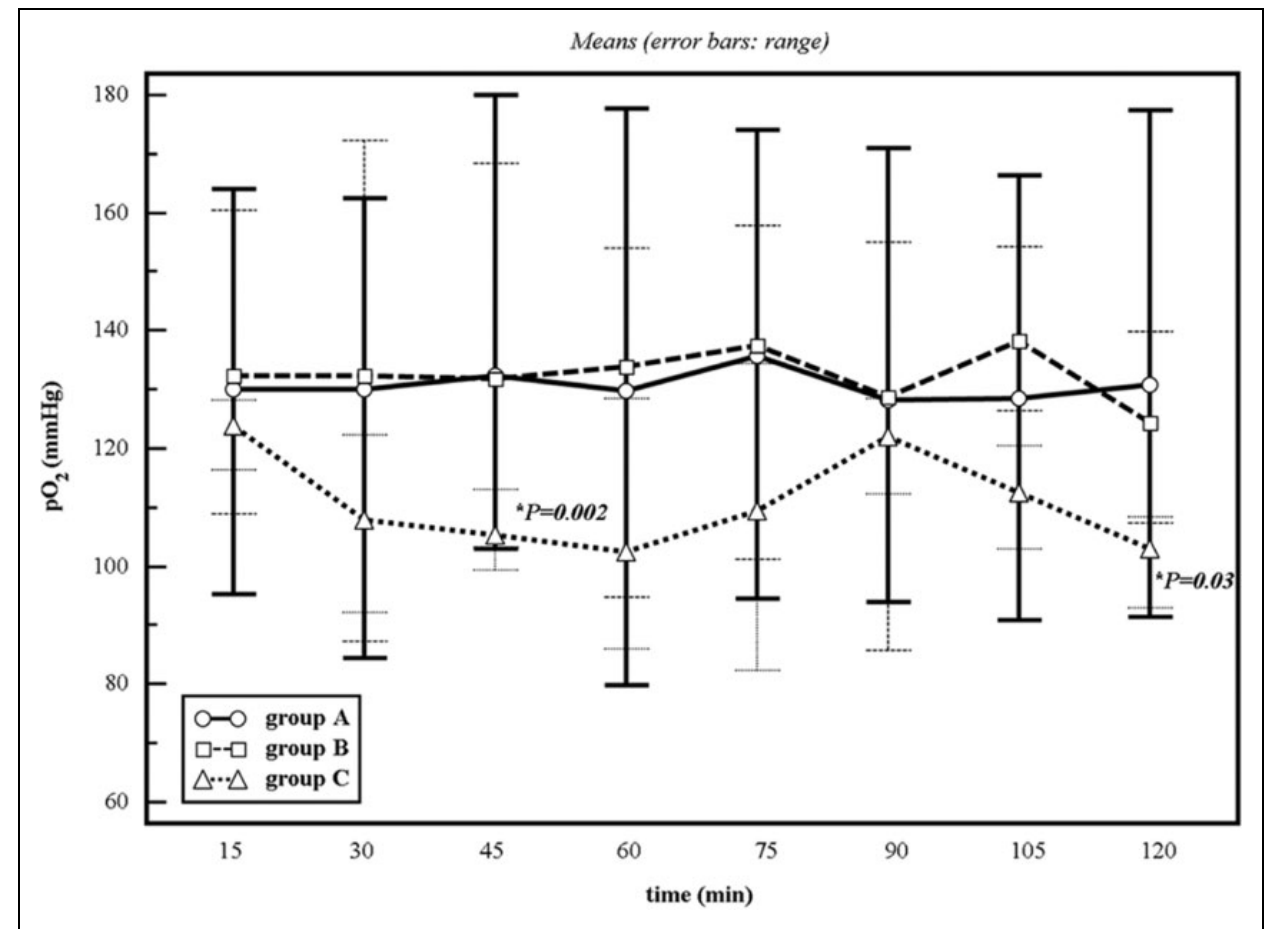

Figure I. Graphical presentation of $\mathrm{pO}_{2}$ pressure (mean values with ranges) in cerebrospinal fluid during the first I20 minutes after aortic clamping in each group; $P$ value: statistical comparison between groups $A$ and $C$.

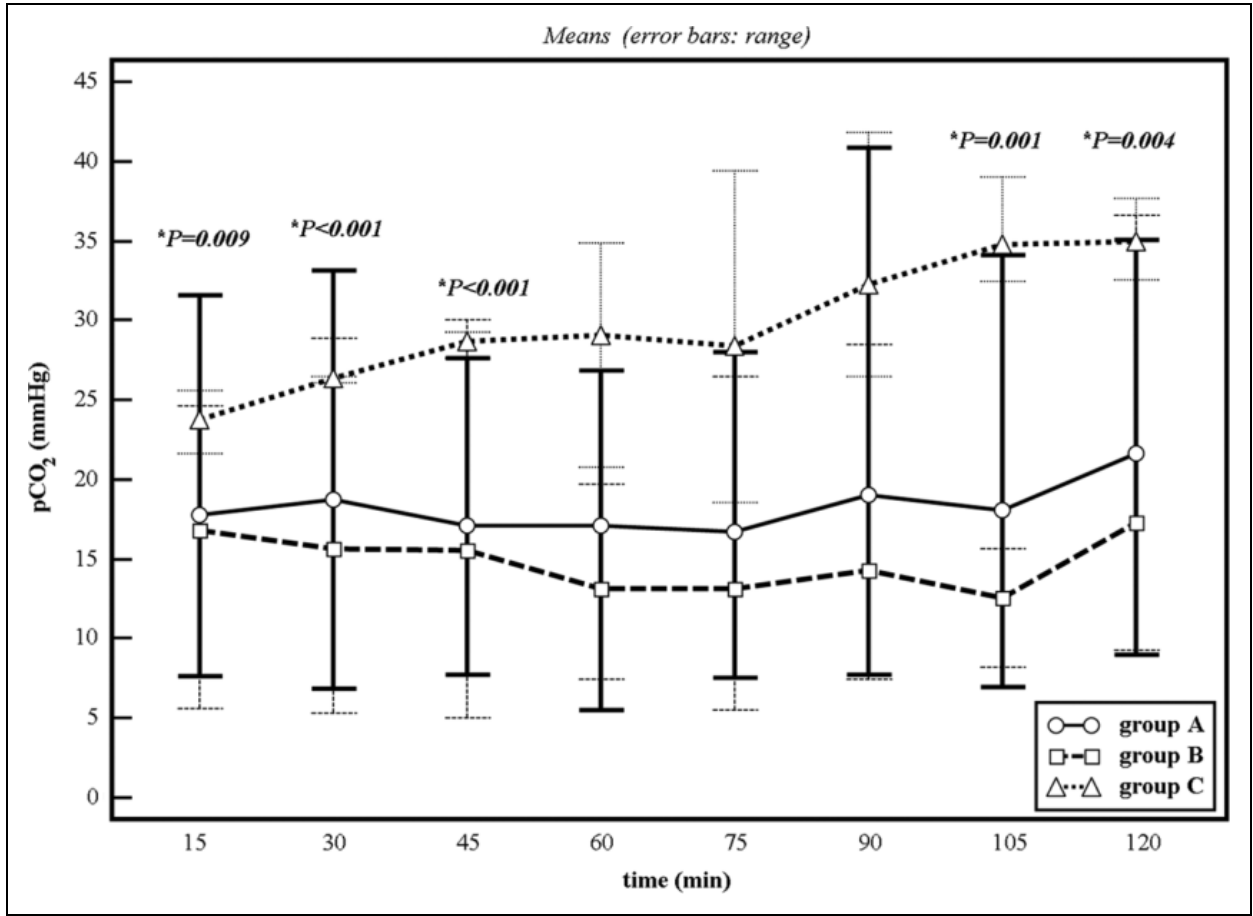

Figure 2. Graphical presentation of $\mathrm{PCO}_{2}$ pressure (mean values with ranges) in cerebrospinal fluid during the first 120 minutes after aortic clamping in each group; $P$ value: statistical comparison between groups $A$ and $C$.

\section{Discussion}

Our proof-of-principle study concerning changes in CSF during TAAA surgery shows that measurement of gas tensions in CSF is feasible and can reflect severe injury such as paraplegia. Nonetheless, (fortunately) only 3 patients reached the end point of paraplegia and showed subsequent significant changes in CSF gas tensions. However, especially for group B, 
Table 3. Mean $\mathrm{pH}$ Values $( \pm \mathrm{SD})$ in Cerebrospinal Fluid From the Beginning (Aortic Clamping) Until the End of Distal Perfusion in Each Group. ${ }^{\text {a }}$

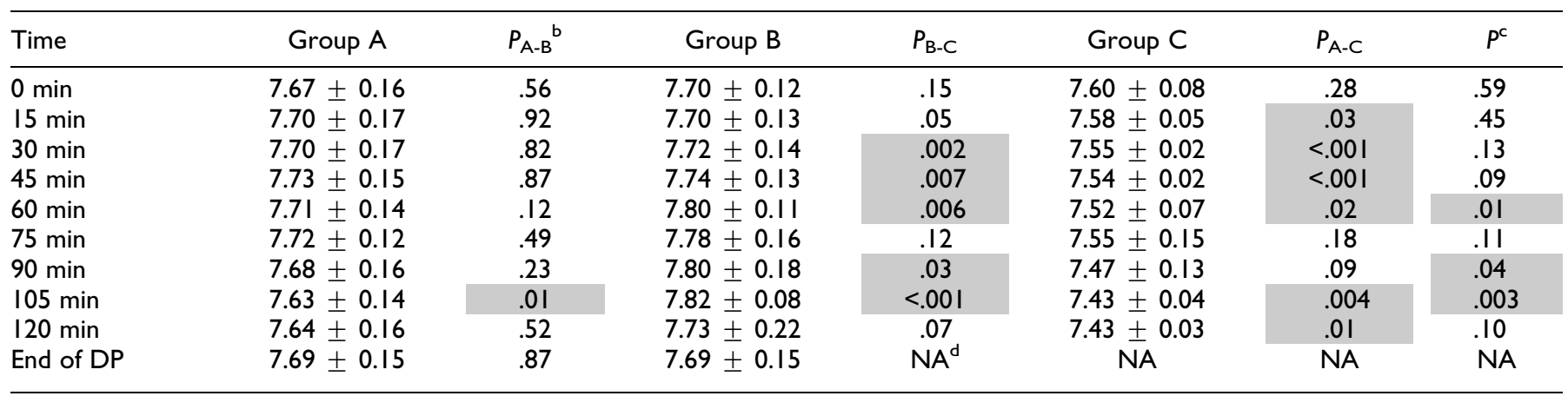

Abbreviations: ANOVA, analysis of variance; DP, distal perfusion; SD, standard deviation.

${ }^{\text {ap }} \mathrm{values}$ showing a statistically significant difference are tinged with gray.

${ }^{\mathrm{b}} P_{X-Y}$ : group $X$ versus group $Y$.

${ }^{c}$ Comparison between 3 groups using one-way ANOVA or Kruskal-Wallis test.

${ }^{\mathrm{d}} \mathrm{No}$ statistical test could be performed because $\mathrm{pH}$ at the end of DP was documented in only one patient in group $\mathrm{C}$.

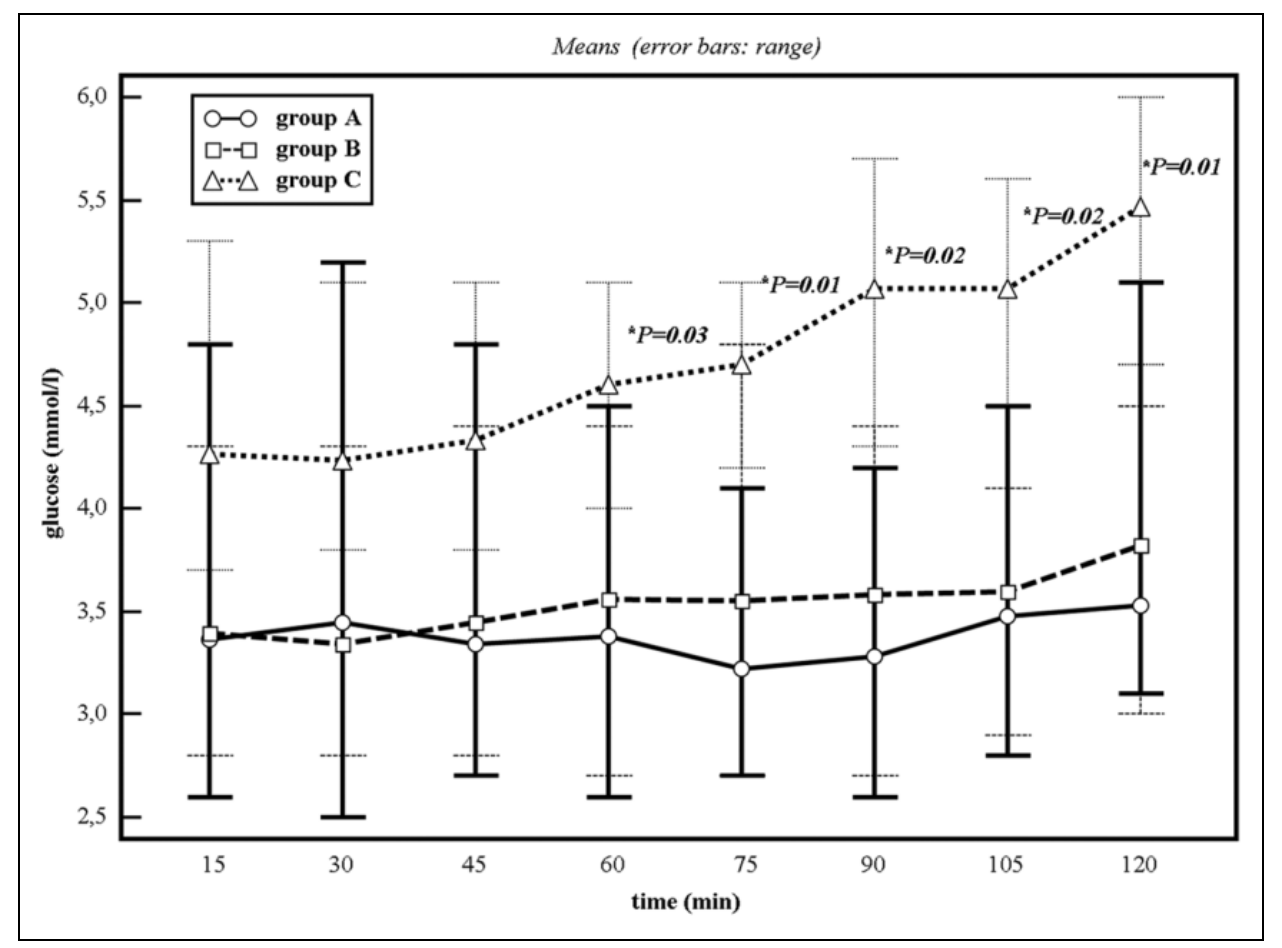

Figure 3. Graphical presentation of glucose (mean values with ranges) in cerebrospinal fluid during the first I 20 minutes after aortic clamping in each group; $P$ value: statistical comparison between groups $A$ and $C$.

subclinical neurological changes occurred in the absence of specific changes in gas tensions, suggesting other mechanism for damage than segmental spinal ischemia. This series demonstrates that besides the much dreaded paraplegia, subclinical damage at the level of the spinal cord also happens in TAAA repair.

The prevention of spinal cord damage supposedly by ischemia during clamping of the aorta in TAAA repair has been a central issue for many years. Several adjuncts, such as DP, have been conclusively shown to reduce this risk significantly on clinical grounds. ${ }^{16}$ Given that the $\mathrm{po}_{2}$ and $\mathrm{pCo}_{2}$ in CSF are directly dependent on the interaction of the perfusate with the active and passive properties of the BBB, they should reflect very accurately the supply situation of the central nervous system near to where the samples of CSF are taken. ${ }^{15}$ The data show that the constituency of the blood, supplying the brain and thus, the choroid plexus, does not correlate well with the gas tensions in CSF, drawn in the lumbar region. In contrast, DP perfusate is directly correlated throughout the perfusion period. This therefore supports DP as an effective adjunct for spinal cord protection in vivo and furthermore supports the use of CSF gas tensions for the assessment of regional spinal perfusion. On the other hand, the number of subclinical neurological deficits observed which have in the past not received such 


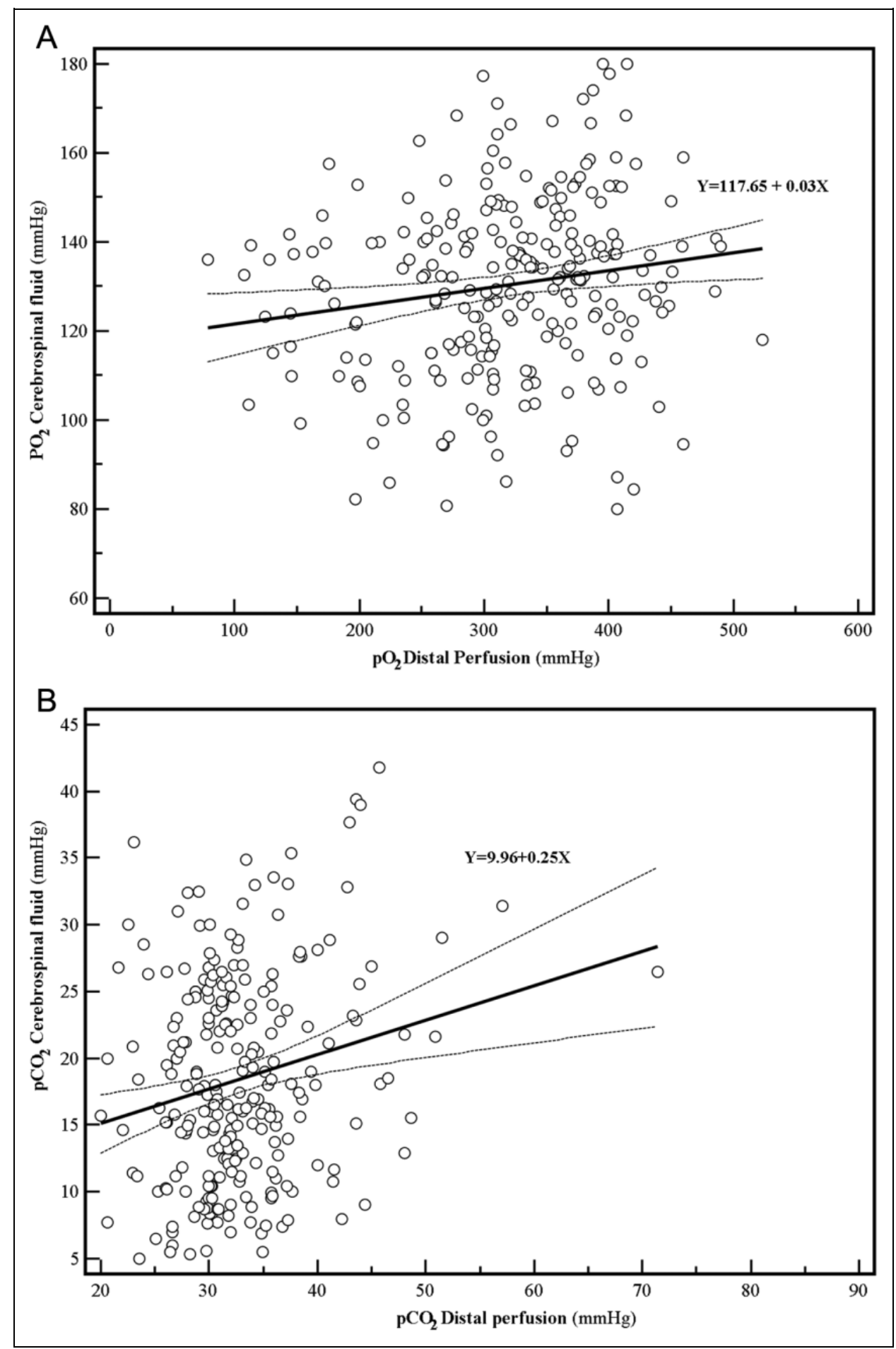

Figure 4. Graphical presentation of the correlation of $\mathrm{pO}_{2}(\mathrm{~A})$ and $\mathrm{pCO}_{2}(\mathrm{~B})$ between distal perfusion and cerebrospinal fluid. The regression line (including regression equation and $95 \%$ confidence interval) illustrates the linear relationship between the respective parameters.

attention and occurred in the absence of specific changes in gas tensions suggests another mechanism for damage than segmental spinal ischemia. Thus, while in the cases of paraplegia relevant changes in $\mathrm{po}_{2}, \mathrm{pCo}_{2}, \mathrm{pH}$, and lactate (the latter in accordance with a previous publication ${ }^{8}$ ) were demonstrated, this was not the case for the patients exhibiting indeed very variable subclinical defects. A mechanism such as microembolization of air or debris would come to mind in analogy to carotid, ${ }^{17}$ cardiac, ${ }^{18}$ or aortic arch surgery. ${ }^{19}$

A further suggestion for a different mechanism causing the 2 clinical pictures comes from the analysis of CSF glucose. Although the glucose ratio between serum and CSF is maintained throughout in all 3 groups, confirming that the overall stabilizing mechanisms of the $\mathrm{BBB}$, as a whole, are 
functioning, the variability in glucose ratio at individual points in time is significantly higher in patients with paraplegia than in the other 2 groups. Thus, the BBB appears to have sustained a serious breach in these cases, which is what one would however also expect to see in minor damage to the spinal cord (possibly less pronounced), which is not the case. However, at this stage, it cannot be ruled out and this is a limitation of this work that here so-called subclinical neurological damages are not due to a separate mechanism but the result of segmental spinal ischemia after all and that the lack of specific changes in the measured parameters are the result of a smaller ischemic event that does not reach the detection threshold of the method. In this case, the, usually, very focal and very diverse nature of the deficits observed remain to be explained however. For the symptomatic group of patients, significant changes happened as early as 30 minutes after clamping but obviously proximal clamping may not be the critical event in every patient. Here the correlation with functional parameters determined online such as evoked potentials will be useful in future studies. Our results are in accordance with an experimental study by Ulus et al showing a gradual and progressive increase in $\mathrm{CSF}_{\mathrm{PCO}_{2}}$ and a decrease in $\mathrm{pH}$ during aortic cross-clamping. ${ }^{20}$

The authors recognize limitations of this exploratory pilot work. The patient number is small with (fortunately) only 3 paraplegias, which, despite the statistical bias, presents a first trend and this is exactly the aim of this work. However, animal studies using a model of spinal ischemia will have to be conducted to further distinguish between spinal cord injury caused my microembolism or segmental ischemia.

\section{Conclusions}

Changes in gas tensions in the CSF in the lumbar region indicate significant changes in the spinal cord supply and correlate well with clinical outcome. Small areas of insufficient supply may go undetected in the clinical course as subclinical neurological deficits and seem to be the result of a different mechanism of injury than regional spinal ischemia. Gas tensions are of no value to detect these. Lumbar CSF constituency best correlates with the DP perfusate and thus, in general, confirms the value of DP for spinal cord protection.

\section{Authors' Note}

Both Maximilian Pichlmaier and Andreas Martens contributed equally to this work.

\section{Declaration of Conflicting Interests}

The author(s) declared no potential conflicts of interest with respect to the research, authorship, and/or publication of this article.

\section{Funding}

The author(s) received no financial support for the research, authorship, and/or publication of this article.

\section{References}

1. Bisdas T, Redwan A, Wilhelmi M, et al. Less-invasive perfusion techniques may improve outcome in thoracoabdominal aortic surgery. J Thorac Cardiovasc Surg. 2010;140(6):1319-1324.

2. Coselli JS, Bozinovski J, LeMaire SA. Open surgical repair of 2286 thoracoabdominal aortic aneurysms. Ann Thorac Surg. 2007;83(2):862-864.

3. Jacobs MJ, de Mol BA, Elenbaas T, et al. Spinal cord blood supply in patients with thoracoabdominal aortic aneurysms. J VasC Surg. 2002;35(1):30-37.

4. Jacobs MJ, Meylaerts SA, de HP, de Mol BA, Kalkman CJ. Strategies to prevent neurologic deficit based on motor-evoked potentials in type I and II thoracoabdominal aortic aneurysm repair. J Vasc Surg. 1999;29(1):48-57.

5. Crawford ES, Svensson LG, Hess KR, et al. A prospective randomized study of cerebrospinal fluid drainage to prevent paraplegia after high-risk surgery on the thoracoabdominal aorta. J Vasc Surg. 1991;13(1):36-45.

6. Etz CD, Luehr M, Kari FA, et al. Paraplegia after extensive thoracic and thoracoabdominal aortic aneurysm repair: does critical spinal cord ischemia occur postoperatively? J Thorac Cardiovasc Surg. 2008;135(2):324-330.

7. Etz CD, Homann TM, Plestis KA, et al. Spinal cord perfusion after extensive segmental artery sacrifice: can paraplegia be prevented? Eur J Cardiothorac Surg. 2007;31(4):643-648.

8. Khaladj N, Teebken OE, Hagl C, et al. The role of cerebrospinal fluid S100 and lactate to predict clinically evident spinal cord ischaemia in thoraco-abdominal aortic surgery. Eur J Vasc Endovasc Surg. 2008;36(1):11-19.

9. van Dongen EP, ter Beek HT, Schepens MA, et al. The relationship between evoked potentials and measurements of S-100 protein in cerebrospinal fluid during and after thoracoabdominal aortic aneurysm surgery. J Vasc Surg. 1999;30(2):293-300.

10. Coselli JS, Tsai PI. Motor evoked potentials in thoracoabdominal aortic surgery: CON. Cardiol Clin. 2010;28(2):361-368.

11. Crawford ES, Mizrahi EM, Hess KR, Coselli JS, Safi HJ, Patel VM. The impact of distal aortic perfusion and somatosensory evoked potential monitoring on prevention of paraplegia after aortic aneurysm operation. J Thorac Cardiovasc Surg. 1988;95(3): 357-367.

12. Gugino LD, Kraus KH, Heino R, et al. Peripheral ischemia as a complicating factor during somatosensory and motor evoked potential monitoring of aortic surgery. J Cardiothorac Vasc Anesth. 1992;6(6):715-719.

13. Jacobs MJ, Mess W, Mochtar B, Nijenhuis RJ, Statius van Eps RG, Schurink GW. The value of motor evoked potentials in reducing paraplegia during thoracoabdominal aneurysm repair. J Vasc Surg. 2006;43(2):239-246.

14. Stadlbauer A, Salomonowitz E, van der RW, Buchfelder M, Ganslandt O. Insight into the patterns of cerebrospinal fluid flow in the human ventricular system using MR velocity mapping. Neuroimage. 2010;51(1):42-52.

15. Ballabh P, Braun A, Nedergaard M. The blood-brain barrier: an overview: structure, regulation, and clinical implications. Neurobiol Dis. 2004;16(1):1-13. 
16. Griepp RB, Griepp EB. Spinal cord perfusion and protection during descending thoracic and thoracoabdominal aortic surgery: the collateral network concept. Ann Thorac Surg. 2007;83(2):865-869.

17. Sharpe RY, Dennis MJ, Nasim A, et al. Dual antiplatelet therapy prior to carotid endarterectomy reduces post-operative embolisation and thromboembolic events: post-operative transcranial Doppler monitoring is now unnecessary. Eur J Vasc Endovasc Surg. 2010;40(2):162-167.

18. Fareed KR, Rothwell PM, Mehta Z, Naylor AR. Synchronous carotid endarterectomy and off-pump coronary bypass: an updated, systematic review of early outcomes. Eur J Vasc Endovasc Surg. 2009;37(4):375-378.

19. Kamiya H, Klima U, Hagl C, et al. Cerebral microembolization during antegrade selective cerebral perfusion. Ann Thorac Surg. 2006;81(2):519-521.

20. Ulus F, Hellberg A, Ulus AT, Karacagil S. Alterations in cerebrospinal fluid $\mathrm{PO}(2), \mathrm{PCO}(2)$, and $\mathrm{pH}$ measurements during and after experimental thoracic aortic cross-clamping. Ann Vasc Surg. 2009;23(1):122-127. doi:10.1016/j.avsg. 2008.06.004. 